\title{
Amalgam as a Filling Material for the Older Person - A Personal Opinion
}

\author{
Jeff Wilson, School of Dentistry, College of Biomedical and Life Sciences, Cardiff \\ University, Wales, UK.
}

Amalgam is a wonderful restorative material that has been used in dentistry for over 150 years. Millions of people world-wide have at least one dental amalgam restoration, and have not suffered any adverse effects. It is easily placed, relatively cheap and durable. It expands while setting thereby creating a tight marginal seal. If moisture is incorporated there will be some increased expansion on setting, but this does not usually cause problems. However, in contrast to tooth coloured restorative materials, it requires mechanical retention to the tooth and more extensive cavity-preparation. Amalgam also is an excellent core material for crowns. When set it is silver in colour, which is unsuitable for anterior teeth. Amalgam fillings can last 40 plus years. They can be repaired easily by cutting a lock into the existing amalgam. Composite restorations, by comparison, are very technique sensitive, not as long lasting as a well-placed amalgam, prone to microleakage and staining and are significantly more expensive. Composites contract on setting and do not bond directly to amalgam so are not the material of choice for repairing defective amalgam margins.

Because amalgam contains toxic mercury there have been calls to ban it from dentistry. Silver-tinmercury amalgam is relatively inert when set, but minute amounts of mercury vaporize due to occlusal wear and when being clinically removed. ${ }^{1,2}$ Mercury vapour is released when mixing and placing amalgam however the risk is negligible, both for dental personnel and patients, and there is no evidence that dental personnel suffer clinical signs of mercury intoxication. ${ }^{3}$ Rarely, some patients experience adverse reactions to amalgam fillings, such as lichenoid reactions in adjacent mucosa or electrolytic reactions with adjacent metal restorations, but there is no evidence that amalgam causes adverse systemic effects in any age group. ${ }^{4}$ The European Commission has therefore concluded that the current use of amalgam poses no health risk beyond occasional, rare local effects. ${ }^{5}$ Dietary intake is the most important source of non-occupational exposure to mercury, and most of the mercury consumed in fish or seafood is methyl mercury. ${ }^{1,6}$ However, the indirect exposure of humans to methyl mercury from dental amalgams is considerably below tolerable limits ${ }^{3}$.

With world-wide efforts to reduce industrial mercury release into the atmosphere there has been growing concern about the release of mercury vapour from human cremations. ${ }^{7}$ Consequently, crematoria in some countries, for example in the European Union, are required to have mercury emission abatement systems to limit the release into the atmosphere, so this is unlikely to create an environmental problem. ${ }^{8,9}$ However, research has shown that the amount of mercury contamination during cremation as a result of amalgam fillings is so low that no additional preventive measures are required. ${ }^{10}$

Composite tooth-coloured materials contain organic substances and undergo chemical reactions within dental cavities and in the adjacent soft tissues during placement, and some of the composite monomers are cytotoxic to pulp and gingival cells in vitro and mutagenic. ${ }^{11}$ Moreover, there have been reports of adverse and allergic reactions to composite resin restorative materials. ${ }^{12-14}$ Unfortunately, information comparing the relative toxicity of tooth coloured restorative materials with amalgam is not available, although we know that amalgam is neither cytotoxic nor mutagenic. ${ }^{4}$

There are occasions when it may not be possible to use tooth coloured restorative materials. Ideally they should be placed with the isolation of a rubber dam, which is not always possible, and application of acidic etchants could be dangerous to some patients for example with dementia, 
severe cognitive disorders or dyskinesia. Moreover, applications of composite resin in small increments for large restorations can be very time consuming and unsuitable for some patients. Older teeth may have little enamel for etching, and the enamel tends to be brittle. ${ }^{15}$ There are occasions when it can be impossible to prevent a cavity flooding with saliva and for this type of patient composite restorations are contra-indicated. In all of these situations amalgam would be the material of choice.

Both dental amalgam and tooth coloured alternative materials are effective and safe to use and they are all associated with very low rates of local adverse effects. ${ }^{11}$ So, let common sense prevail there is no evidence to support a total ban of amalgam in dentistry and there are certain situations where its use would have the best prognosis. As dentists we need a variety of restorative materials and to my mind, until we find something better to replace it, amalgam is essential.

Jeff Wilson BDS, MSc, PhD, DDPH, FDSRCS, Dip Biomed Meth, FHEA

Clinical Senior Lecturer / Consultant in Restorative Dentistry

Specialist in Prosthodontics and in Special Care Dentistry

School of Dentistry

College of Biomedical and Life Sciences

Cardiff University

Wales, U.K.

\section{References}

1. World Health Organization. Inorganic mercury. International Programme on Chemical Safety, Geneva. 1991

2. Ritchie KA, Burke FJT, Gilmour WH, et al. Mercury vapour levels in clinical practices and body levels of dentists and controls, Br Dent J 2004; 197:625-32

3. Scientific Committee on Emerging and Newly Identified Health Risks. 2008. Available at: http://ec.europa.eu/health/ph_risk/committees/04_scenihr_o_016.pdf section3.4.10: pp53

4. Scientific Committee on Emerging and Newly Identified Health Risks. 2008. Available at: http://ec.europa.eu/health/ph_risk/committees/04_scenihr_o_016.pdf section 4.2.1: pp55

5. European Commission. Tooth filling materials: dental amalgams and alternative materials. 2008. Available at: http://ec.europa.eu/health/opinions/en/dental-amalgam/ section 4.2

6. World health Organization. Methylmercury. International Programme on Chemical Safety, Geneva. 1990

7 Künzler P, Andrée M. More mercury from crematoria, Nature 1991; 349:746-7

8 Limit values and quality objectives for mercury discharge by sectors other than the chloralkali industry. European Commission Directive EC 84/156/EEC 1984.

$9 \quad$ Robinson L. Amalgam and crematoria, Br Dent J 2012; 212:57 
10 Matter-Grütter C, Baillod R, Imfield T, Lutz F. Mercury emission measurements in a crematorium - the dentistry aspects, Schweiz Montatsschr Zahnmed 1995; 105:1023-28

11 Scientific Committee on Emerging and Newly Identified Health Risks. 2008. Available at: http://ec.europa.eu/health/ph_risk/committees/04_scenihr_o_016.pdf section3.4.10: pp52

12 Lind PO. Oral lichenoid reactions related to composite restorations. Preliminary report, Act Odont Scand 1988; 46:63-65

13 Nathanson D, LockhartP. Delayed extra-oral hypersensitivity to dental composite material, Oral Surg Oral Med Oral Pathol 1979; 47:329-33

14 Schurs AH, van Amerongen HP. General health damage by restorations of composite, Ned Tijdschr Tandheelkd 1996; 103:444-47

15 Ketterl W. Age-induced changes in the teeth and their attachment apparatus, Int Dent J 1983;33:262-63 